\title{
Allyl isothiocyanate inhibits cell metastasis through suppression of the MAPK pathways in epidermal growth factor-stimulated HT29 human colorectal adenocarcinoma cells
}

\author{
KUANG-CHI LAI ${ }^{1,3 *}$, CHI-CHENG LU ${ }^{2,4 *}$, YIH-JING TANG ${ }^{5,6}$, JO-HUA CHIANG $^{2,7}$, \\ DAIH-HUANG KUO ${ }^{8}$, FU-AN CHEN $^{8}$, I-LI CHEN ${ }^{8}$ and JAI-SING YANG ${ }^{2}$
}

${ }^{1}$ School of Medicine, ${ }^{2}$ Department of Pharmacology, China Medical University, Taichung 404; ${ }^{3}$ Department of Surgery, China Medical University Beigang Hospital, Yunlin $651 ;{ }^{4}$ Department of Food Science and Biotechnology, National Chung Hsing University, Taichung 402; ${ }^{5}$ Department of Family Medicine, Taichung Veterans General Hospital, Taichung 406; ${ }^{6}$ School of Medicine, Chung Shan Medical University, Taichung 402;

${ }^{7}$ Department of Chemistry, National Cheng Kung University, Tainan $701 ;{ }^{8}$ Department of Pharmacy and Graduate Institute of Pharmaceutical Technology, Tajen University, Pingtung 907, Taiwan, R.O.C.

Received September 14, 2013; Accepted November 7, 2013

DOI: $10.3892 / o r .2013 .2865$

\begin{abstract}
Allyl isothiocyanate (AITC) has been found to present sources from consumed cruciferous vegetables. AITC is known to possess pharmacological and anticancer activities. The present study was designed to test the hypothesis that AITC suppressed the invasion and migration of epidermal growth factor (EGF)-stimulated HT29 cells and to elucidate the mechanisms for the antimetastatic abilities in vitro. The invasion and migration of EGF-stimulated HT29 cells were determined individually by Transwell cell invasion and wound-healing assays. Our results showed that AITC effectively inhibited both the invasive and migratory ability of HT29 cells. Furthermore, AITC downregulated the protein levels of matrix metalloproteinase-2 (MMP-2), MMP-9 and mitogen-activated protein kinases (MAPKs) (p-JNK, p-ERK and p-p38) by western blot analysis in HT29 cells following EGF induction. Thus, the metastatic responses in AITCtreated HT29 cells after EGF stimulation were mediated by the MMP-2/-9 and MAPK signaling pathways. We also used gene expression microarrays to investigate the gene levels related to cell growth, G-protein coupled receptor, angiogenesis, cell adhesion, cell cycle and mitosis, cell migration, cytoskeleton organization, DNA damage and repair, transcription and
\end{abstract}

Correspondence to: Dr Jai-Sing Yang, Department of Pharmacology, China Medical University, 91 Hsueh-Shih Road, Taichung 404, Taiwan, R.O.C.

E-mail: jaising@mail.cmu.edu.tw

*Contributed equally

Key words: allyl isothiocyanate, metastasis, mitogen-activated protein kinase, DNA microarray, epidermal growth factor, colorectal adenocarcinoma cells translation, EGFR and PKB/mTOR signals. In summary, it is possible that AITC suppresses the invasion and migration of EGF-induced HT29 cells, resulting from MMP-2/-9 and MAPKs. Hence, AITC may be beneficial in the treatment of human colorectal adenocarcinoma in the future.

\section{Introduction}

Allyl isothiocyanate (AITC) is a cancer chemopreventive phytochemical agent found in naturally occurring dietary isothiocyanates (ITCs) (1-3). AITC was found to have a major glucosinolate in several commonly consumed cruciferous vegetables (cabbage, cauliflower and kale as well as Brussels sprouts) (4,5). Previous studies showed that AITC inhibited the growth of various human cancer cell lines, such as colorectal carcinoma $(5,6)$, lung cancer $(7)$, leukemia (4), breast adenocarcinoma (8), bladder cancer $(5,9)$, brain malignant glioma $(10)$, neuroblastoma $(11)$, hepatoma $(12,13)$ and prostate cancer cells $(2,14,15)$. Of note, the half maximal inhibitory concentration $\left(\mathrm{IC}_{50}\right)$ values for the anticancer cell growth appear at the low micromolar ranges of AITC (9). On the other hand, AITC appears to be significantly less toxic to normal human bladder epithelial cells $(5,9)$. AITC is likely to attenuate tumor cell growth by causing cell cycle arrest $(8,10)$, induction of cell apoptosis $(1,2)$ and to suppress metastasis via inhibition of invasion and migration in neoplastic cells $(5,16)$. The earlier studies in our laboratory also demonstrated that AITC triggered G2/M phase arrest and provoked apoptosis in MDA-MB-468 human breast adenocarcinoma cells (8) and GBM 8401 human brain glioblastoma multiforme cells (10).

Matrix metalloproteinases (MMPs) play key roles during cancer cell metastasis $(5,16)$. The levels of cell adhesion, invasion and migration were suppressed through the transcription of MMP-2/-9 by AITC at 0.1-5 $\mu \mathrm{M}$ in SK-Hep-1 human liver cancer cells (16). However, few studies have addressed the AITC-inhibited cell metastasis in epidermal growth factor (EGF)-stimulated HT29 human colorectal cancer cells. The 
aim of the present research was to determine the decrease of cell metastasis in EGF-treated HT29 cells by AITC. The present study indicated that AITC suppressed the extracellular signal-regulated kinase (ERK), p38, c-Jun $N$-terminal kinase (JNK) mitogen-activated protein kinase (MAPK) pathways and, thus, reduced the MMP-2 and -9, leading to the inhibition of metastatic effects in EGF-stimulated HT29 cells in vitro. Furthermore, we examined the antimetastatic effects of AITC by altering the cell cycle response-related gene expression utilizing DNA microarray analysis. Our study is the first to report that MAPK signals are pivotal for the antimetastatic response of HT29 human colorectal adenocarcinoma cells induced by AITC.

\section{Materials and methods}

Materials and reagents. RPMI-1640 medium, fetal bovine serum (FBS), penicillin/streptomycin and trypsin-EDTA were bought from Gibco by Life Technologies (Carlsbad, CA, USA). Millicell Hanging Cell Culture Inserts (polyethylene terephthalate filters with $8 \mu \mathrm{m}$ pore size) were purchased from Merck Millipore Corp. (Billerica, MA, USA). All primary antibodies for immunoblotting and horseradish peroxidase (HRP)-conjugated secondary antibodies were purchased from Santa Cruz Biotechnology, Inc. (Santa Cruz, CA, USA). AITC and all other chemicals were purchased from Sigma-Aldrich Corp. (St. Louis, MO, USA) unless otherwise specified.

Cell culture. The human colorectal adenocarcinoma HT29 cell line was obtained from the Bioresource Collection and Research Center (BCRC) of Food Industry Research and Development Institute (Hsinchu, Taiwan). Cells were plated into $75-\mathrm{cm}^{2}$ tissue culture flasks and cultured in RPMI-1640 medium with $10 \% \mathrm{FBS}, 100 \mathrm{U} / \mathrm{ml}$ penicillin and $100 \mu \mathrm{g} / \mathrm{ml}$ streptomycin in a humidified atmosphere of $5 \% \mathrm{CO}_{2}$ and $95 \%$ air at $37^{\circ} \mathrm{C}$ as previously described $(17,18)$. Cells were detached by $0.25 \%$ trypsin/0.02\% EDTA to keep cell growth.

Transwell cell invasion assay. HT29 cells at a density of $1 \times 10^{4}$ cells/0.4 ml serum-free RPMI-1640 medium were seeded with or without (basal) $10 \mathrm{ng} / \mathrm{ml}$ EGF into the upper chambers (Millicell Hanging Cell Culture Inserts; Merck Millipore Corp.) after pre-coating with Matrigel (BD Biosciences, San Jose, CA, USA) in the presence or absence of AITC at 5 and $10 \mu \mathrm{M}$. Each lower chamber was filled with $600 \mu \mathrm{l}$ of $10 \% \mathrm{FBS}$ medium. After incubation for $24 \mathrm{~h}$, the chambers were removed from the wells to measure invasive ability and then fixed with methanol for $15 \mathrm{~min}$ before the noninvasive cells were wiped with a cotton swab. Consequently, cells were stained with $2 \%$ crystal violet for $10 \mathrm{~min}$, and the invaded cells were then photographed under a phase-contrast microscope. The number of invasive cells was presented in the membrane/filter of three random fields as previously described $(19,20)$. The invasion assay was performed with a $100 \%$ support value of basal cells, and invasive cells were quantified using NIH ImageJ 1.47 program.

Wound healing assay. HT29 cells in 6 -well plates $\left(\sim 5 \times 10^{5}\right.$ cells/well) were grown to $80 \%$ confluence, and cell monolayer was scratched by a $200-\mu 1$ pipette tip to create a gap of constant width. Subsequently, cells were washed with PBS twice to remove floating cells. Cells were exposed in the presence or absence of $10 \mathrm{ng} / \mathrm{ml}$ EGF and then treated with or without 5 and $10 \mu \mathrm{M}$ of AITC in serum-free RPMI-1640 medium for up to $24 \mathrm{~h}$. The number of migrating cells in the gap was captured under a phase-contrast microscope as described elsewhere $(21,22)$. Images for the scratch area of each well were counted in three random fields from each triplicate treatment. The number of migrated cells in EGF-untreated cells (basal) was expressed at 100\% and these in treated cells showed related to the basal cells.

Total protein extraction and western blot analysis. HT29 cells were incubated with or without $10 \mathrm{ng} / \mathrm{ml}$ EGF and individually exposed to 5 and $10 \mu \mathrm{M}$ of AITC. After a 24-h treatment, each whole-cell protein extract was harvested and lysed in the PRO-PREP protein extraction solution (Intron Biotechnology, Seongnam-si, Gyeonggi-do, Korea). The protein concentration of the cell lysate was estimated with the Bio-Rad protein assay kit (Bio-Rad Laboratories, Hercules, CA, USA), and the total proteins $(40 \mu \mathrm{g})$ were electrophoresed by $10-12 \%$ SDS-PAGE before being transferred and blotted using iBlot dry blotting system with polyvinylidene difluoride (PVDF) membrane (Invitrogen by Life Technologies). The membranes were blocked and probed first with specific antibodies in blocking buffer at $4^{\circ} \mathrm{C}$ overnight as previously described $(23,24)$, followed by incubation with the appropriate horseradish peroxide (HRP)-linked secondary antibodies. Immobilon Western Chemiluminescent HRP substrate (Millipore) and X-ray film (GE Healthcare, Piscataway, NJ, USA) were applied to visualize, and the membranes were stripped and reprobed with $\beta$-actin to normalize to the protein expression as described elsewhere (25).

RNA extraction and expression microarray. HT29 cells $\left(1 \times 10^{6}\right.$ cells/well) were maintained in RPMI-1640 medium with $10 \mathrm{ng} / \mathrm{ml}$ EGF and treated with or without $10 \mu \mathrm{M}$ AITC for $24 \mathrm{~h}$. After exposure, cell pellets were subsequently harvested, and the total RNA from each treatment was purified using the Qiagen RNeasy Mini kit (Qiagen, Valencia, CA, USA) as previously described $(23,26)$. The RNA purity was determined to check the quality at $260 \mathrm{~nm}$ and $280 \mathrm{~nm}$ using a NanoDrop 1000 Spectrophotometer (Thermo Fisher Scientific, Wilmington, DE, USA) (26,27). Each sample (300 ng) was amplified and labeled using the GeneChip WT Sense Target Labeling and Control Reagents kit (Affymetrix, Santa Clara, CA, USA) for expression analysis. The synthesized cDNA was labeled with fluorescence, and then hybridized for $17 \mathrm{~h}$ at $45^{\circ} \mathrm{C}$ and $60 \mathrm{rpm}$ using Affymetrix GeneChip Human Gene 1.0 ST array (Affymetrix) to determine microarray hybridization following the manufacturer's recommendations.

The arrays were subsequently washed by Fluidics Station 450 (Affymetrix) and stained with streptavidin-phycoerythrin (GeneChip Hybridization, Wash and Stain kit, Affymetrix), and were scanned on a GeneChip Scanner 3000 (Affymetrix). The localized concentrations of fluorescent molecules were quantitated and analyzed using Expression Console software (Affymetrix) with default RMA parameters as previously described $(28,29)$. The gene expression level of a 1.8-fold-change altered by AITC was considered a difference. 
A

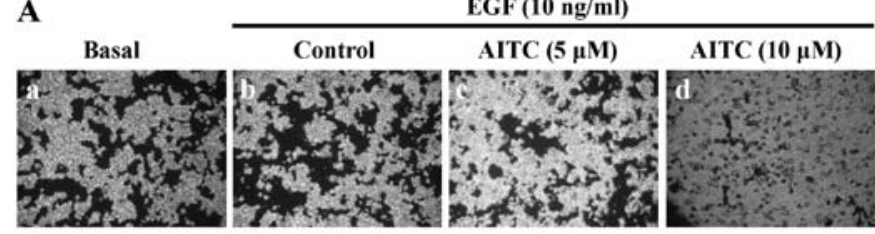

\section{B}

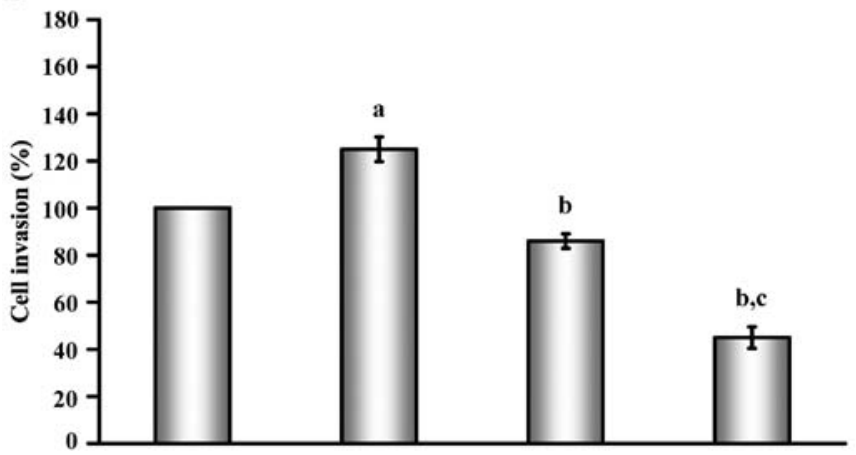

Figure 1. AITC suppresses cell invasion of EGF-induced HT29 cells. Cells in the presence or absence $10 \mathrm{ng} / \mathrm{ml}$ EGF were treated with or without 5 and $10 \mu \mathrm{M}$ of AITC for $24 \mathrm{~h}$. (A) Cell invasion was examined by Boyden chamber assay, and the polycarbonate membranes (pore size, $8 \mu \mathrm{m}$ ) were precoated with Matrigel prior to use. (B) Invasive ability of HT29 cells was quantified by counting the number of cells under microscopy. The reported values are presented as the means $\pm \mathrm{SD}(\mathrm{n}=3) .{ }^{\mathrm{a}} \mathrm{P}<0.05$, when compared with basal sample; ${ }^{\mathrm{b}, \mathrm{P}} \mathrm{P}<0.05$, significant difference compared with control and $5 \mu \mathrm{M}$ AITC treatment in HT29 cells after EGF challenge, respectively.

Statistical analysis. Each experiment was performed in triplicate and expressed as the means \pm standard deviation (SD) of analysis. The results were assessed by one-way ANOVA followed by Bonferroni's multiple comparison test, and the value of $\mathrm{P}<0.05$ indicated statistically significant differences from other treatments.

\section{Results}

AITC suppresses EGF-stimulated invasion of HT29 cells. To investigate the effects of AITC on HT29 cell invasion in vitro, Transwell cell invasion assay was performed to determine the invasive ability of the HT29 cells with or without EGF stimulation. Results in Fig. 1A show that AITC at 5 and $10 \mu \mathrm{M}$ markedly decreased the invasion of HT29 cells induced by EGF relative to the control group. In addition, the invasive ability was reduced in a concentration-dependent manner (Fig. 1B). Our data demonstrated that AITC may effectively inhibit the invasion of HT29 cells in vitro.

AITC inhibits EGF-triggered HT29 cell migration. As shown in Fig. 2, using the wound healing assay, the concentrations of 5 and $10 \mu \mathrm{M}$ of AITC markedly inhibited HT29 cell migration (Fig. 2A) by $\sim 48$ and $81 \%$, respectively, (Fig. 2B) after a 24-h incubation when compared with EGF-induced migration of HT29 cells. Based on these findings, we concluded that the migratory ability occurred in AITC-treated HT29 cells.

AITC alters the abundance of protein level with metastatic response in HT29 cells. Next, we clarified if AITC-suppressed migration and invasion is mediated through downregulation

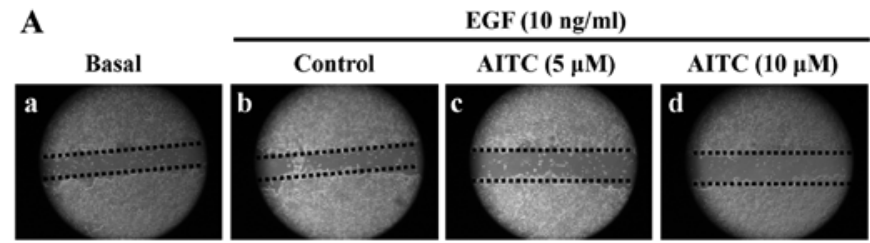

B

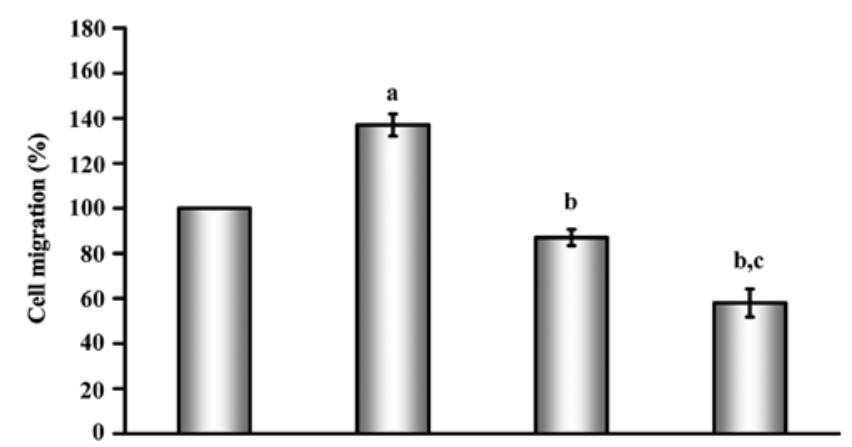

Figure 2. AITC reduces the migration of EGF-stimulated HT29 cells Cells were exposed to 5 and $10 \mu \mathrm{M}$ of AITC for $24 \mathrm{~h}$ after $10 \mathrm{ng} / \mathrm{ml} \mathrm{EGF}$ induction. (A) The wound healing assay was carried out to evaluate the inhibitory effects of AITC on HT29 cell migration. (B) The quantitation of the migrated cells in the gap of constant width was performed, and the HT-29 cells without EGF treatment (basal) were defined as $100 \%$. Results shown as means $\pm \mathrm{SD}(\mathrm{n}=3) .{ }^{\mathrm{a}} \mathrm{P}<0.05$, statistical comparison of the basal group; ${ }^{\mathrm{b}, \mathrm{c}} \mathrm{P}<0.05 \mathrm{vs}$. control and $5 \mu \mathrm{M}$ AITC treatment of EGF-treated HT29 cells, respectively.

of associated protein signals. Treatment of EGF-treated HT29 cells with 5 and $10 \mu \mathrm{M}$ of AITC for $24 \mathrm{~h}$, and data shown in Fig. 3 revealed that AITC decreased the protein expressions of MMP-2 and MMP-9 in EGF-treated HT29 cells. Alternatively, the level of the tissue inhibitor of metalloproteinase-1 (TIMP-1) was increased in examined HT29 cells (Fig. 3). We further determined the effect of AITC on the MAPK signaling pathways. Our results indicated that AITC inhibited the phosphorylation of JNK, ERK and p38 signaling in HT29 cells after EGF exposure (Fig. 3). Based on these results, we found that AITC affected MMP-9/-2, TIMP-1 and MAPKs signaling in EGF-treated HT29 cells in vitro.

Microarray analysis. Data were analyzed to examine the expressed genes in EGF-stimulated HT29 cells treated with or without AITC as can be seen in Table I. We showed that the transcripts of 58 genes were upregulated, while these of 24 genes were downregulated in HT29 cells exposed to AITC after EGF induction, respectively. Our results revealed that AITC regulated the expression of important genes that control cell growth (AKR1C2, AKR1C1, AKR1C1, ALDH3A1, TXNRD1 and ANKRD11), G-protein coupled receptor (AKR1C1 and AKR1C3), angiogenesis (HMOX1, MT1G and VEZF1), cell adhesion (TROAP, ITGB1 and EZR), cell cycle and mitosis (CSNK1A1, CDC20, BIRC5, KIF20A, CSNK1A1, ITGB1 and LIMA1), cell migration (HMOX1 and ITGB1), cytoskeleton organization (KIF20A and LIMA1), DNA damage and repair (SRXN1, G6PD, PTGR1, UNG, USP10 and PRKDC) and transcription and translation (EIF4A1, TRIM16 and ZKSCAN1) as listed in Table I. The Gene to GO BP test 


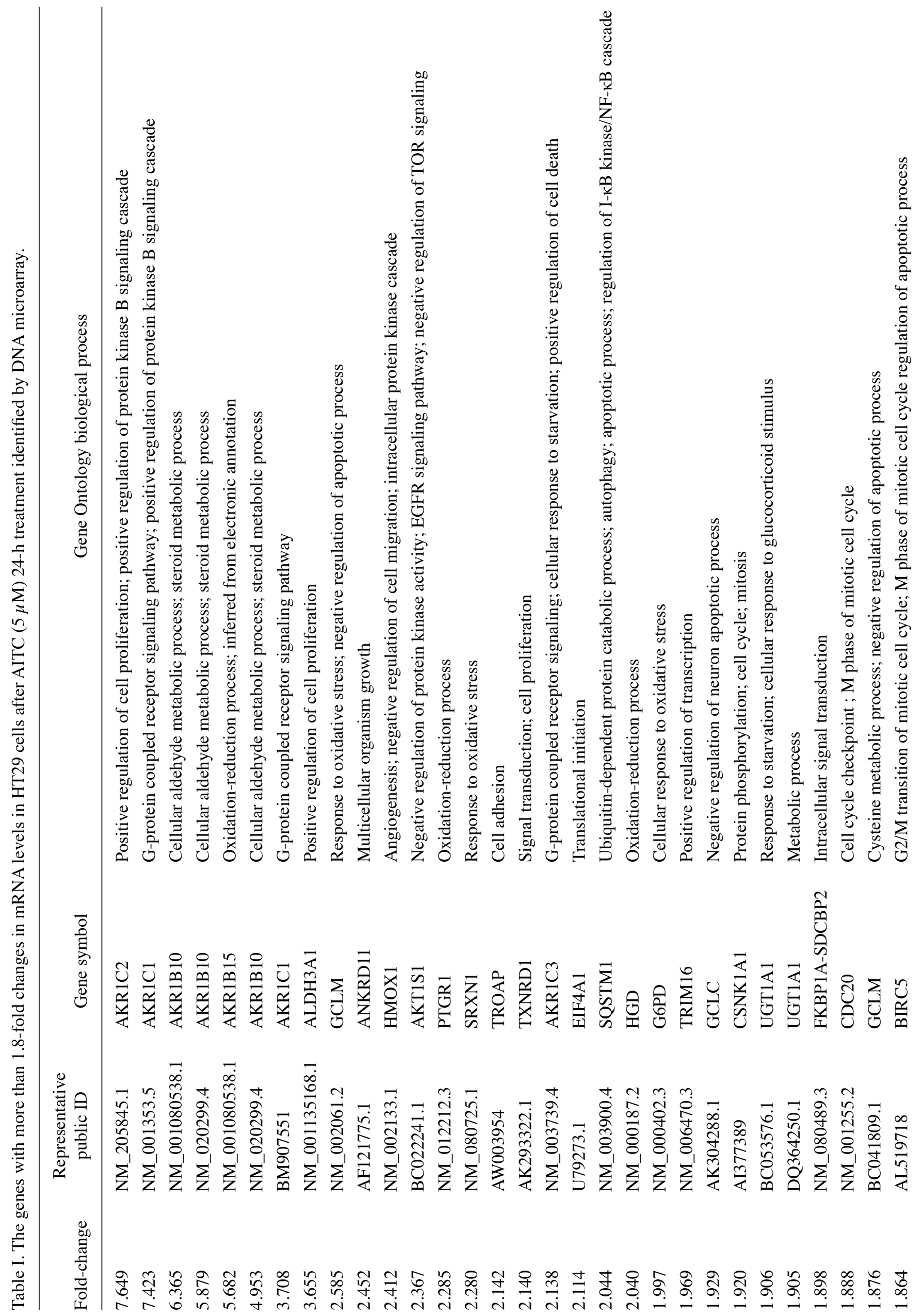




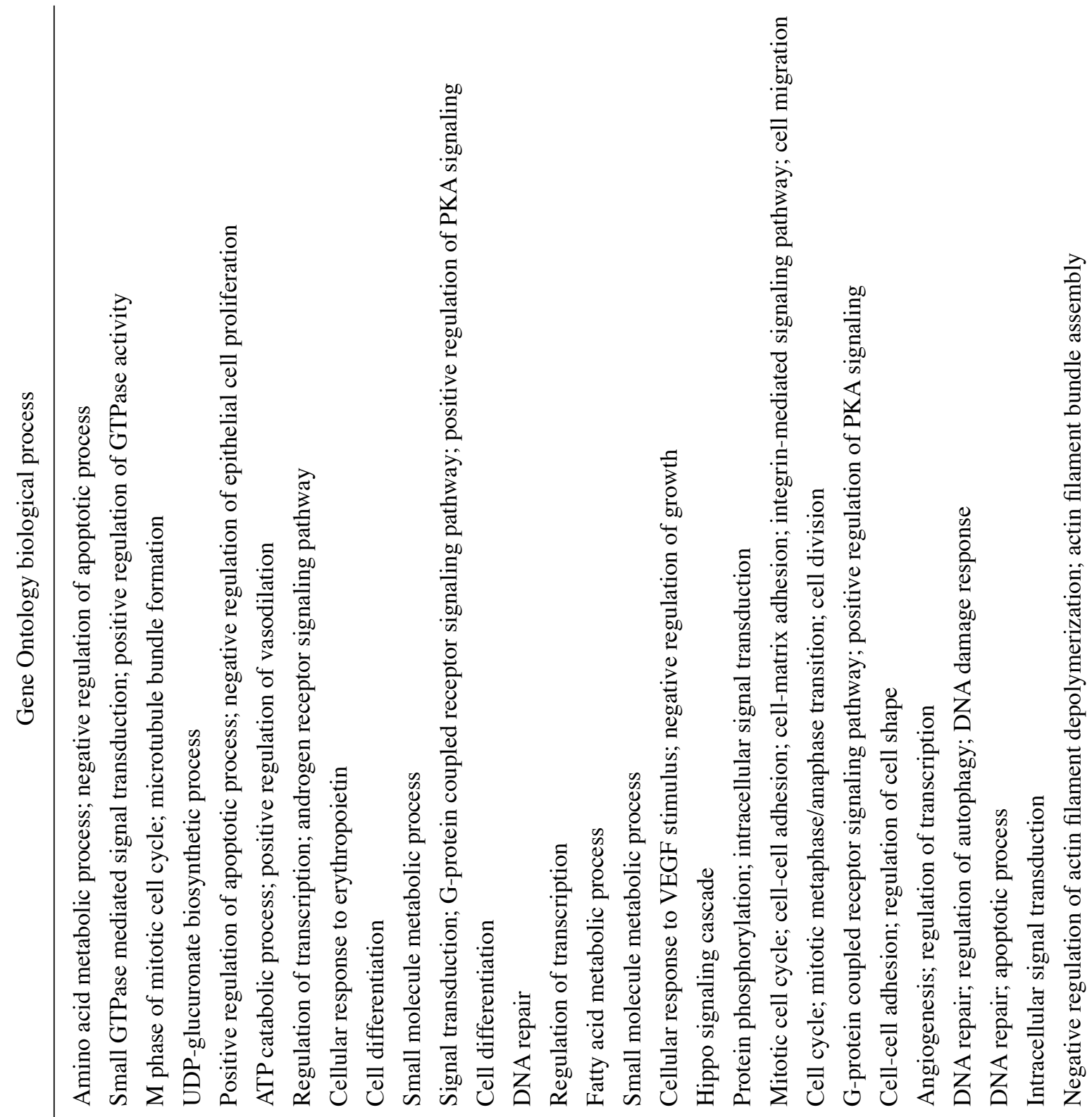

章

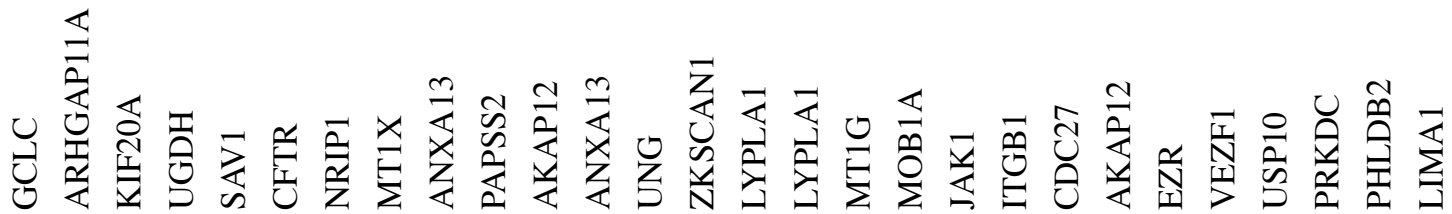

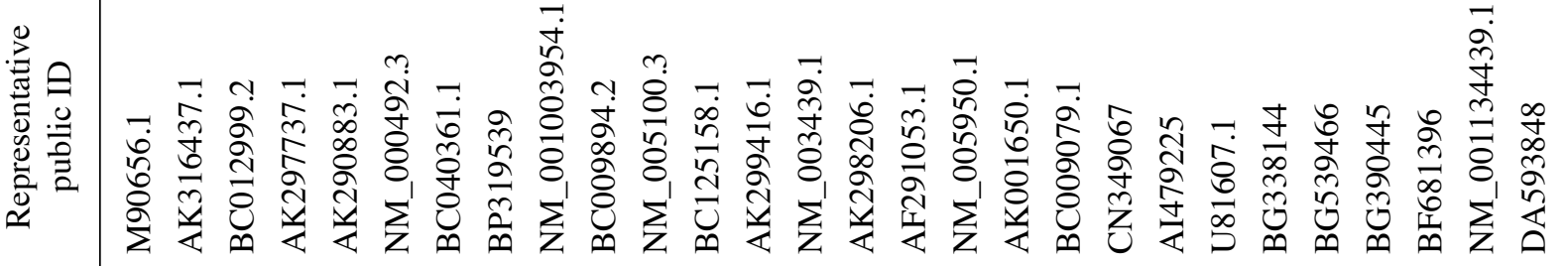


Table II. Gene to GO BP test for over-representation in HT29 cells after AITC (5 $\mu \mathrm{M})$ 24-h treatment identified by DNA microarray.

\begin{tabular}{lccc}
\hline Term & Count & Percentage & P-value \\
\hline GO:0007049-cell cycle & 114 & 10.73446 & $1.61 \mathrm{E}-18$ \\
GO:0000278-mitotic cell cycle & 69 & 6.497175 & $2.74 \mathrm{E}-16$ \\
GO:0022403-cell cycle phase & 73 & 6.873823 & $6.45 \mathrm{E}-16$ \\
GO:0022402-cell cycle process & 87 & 8.19209 & $3.02 \mathrm{E}-15$ \\
GO:0048285-organelle fission & 50 & 4.708098 & $9.32 \mathrm{E}-15$ \\
GO:0000280-nuclear division & 48 & 4.519774 & $3.52 \mathrm{E}-14$ \\
GO:0007067-mitosis & 48 & 4.519774 & $3.52 \mathrm{E}-14$ \\
GO:0000087-M phase of mitotic cell cycle & 48 & 4.519774 & $7.18 \mathrm{E}-14$ \\
GO:0000279-M phase & 60 & 5.649718 & $7.22 \mathrm{E}-14$ \\
GO:0051301-cell division & 54 & 5.084746 & $1.30 \mathrm{E}-12$ \\
\hline
\end{tabular}

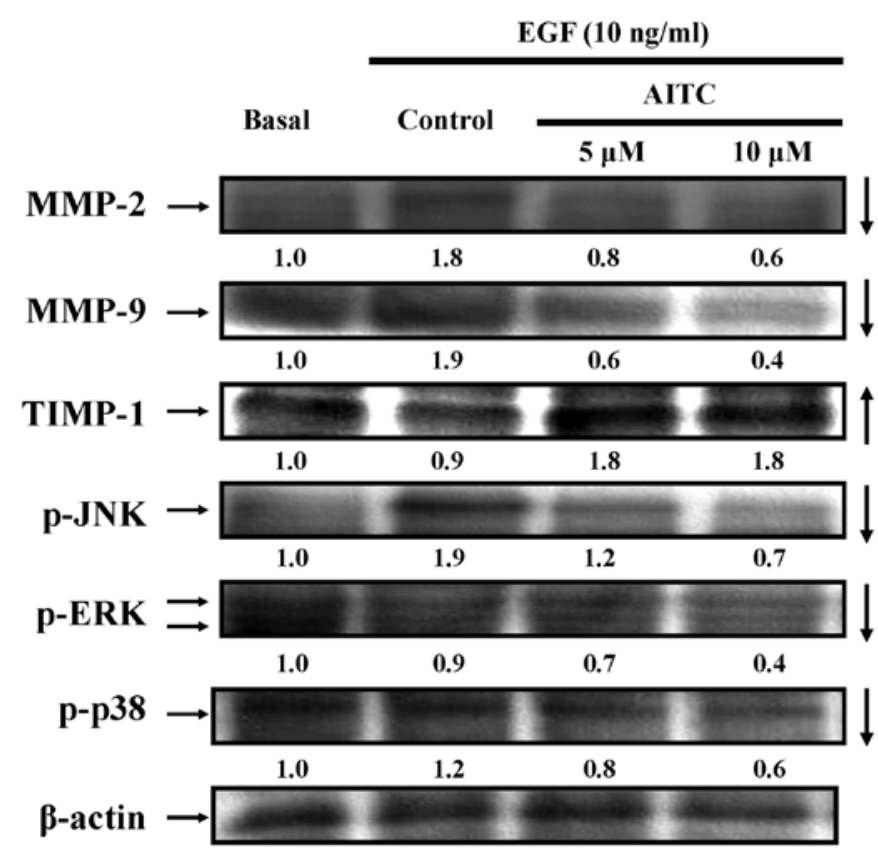

Figure 3. AITC alters the matrix metalloproteinases (MMPs) and mitogen-activated protein kinases (MAPKs) associated protein expression of EGF-treated HT29 cells. Cells were exposed to $10 \mathrm{ng} / \mathrm{ml}$ EGF and then incubated with or without 5 and $10 \mu \mathrm{M}$ of AITC for the indicated time $(24 \mathrm{~h})$. Whole-cell lysates were subjected to immunoblotting as described in Materials and methods The protein expression of MMP-2, -9, tissue inhibitor of metalloproteinase 1 (TIMP-1), phosphorylated c-Jun $N$-terminal kinase (p-JNK), phosphorylated extracellular regulated protein kinase (p-ERK) and phosphorylation of p38 (p-p38) was probed using specific antibodies. $\beta$-actin protein level was adjusted for equivalent loading. Representative data are shown in three separate experiments with similar results.

for over-representation and pathways in HT29 cells after AITC 24 h-treatment identified by DNA microarray are listed in Table II. AITC altered the expression of negative regulation of protein kinase activity on EGFR and PKB/mTOR signaling genes (AKR1C2, AKR1C1, AKR1C1 and AKT1S1) in examined HT29 cells.

\section{Discussion}

It is well documented that cell metastasis is one of the major causes of colorectal cancer-related mortality $(17,18,30)$. Interference with human epidermal growth factor receptor (EGFR) or downstream intracellular signaling provides a novel approach in cancer chemotherapeutic agents (31-33). Activation of the EGFR pathway promotes by EGF-stimulated responsible for colorectal cancer cell growth, invasion, metastasis and inhibition of cell death $(34,35)$. Following EGF stimulation, EGFR is autophosphorylated, which turns on downstream intracellular signaling cascades such as MAPKs (ERK, p38 and JNK) signaling pathways $(36,37)$. In the present study, we found that AITC was able to inhibit the invasion and migration in EGF-stimulated HT29 cells and could have a potential to treat colorectal cancer cell metastasis (Figs. 1 and 2). Cancer metastasis is a complicated progression that involves the increase in cell invasion, migration and degradation of extracellular matrix (ECM) then circulation in the vascular and lymphatic systems, finally the residence in distant organs $(5,16,38)$. The matrix metalloproteinases (MMPs) are responsible for ECM degradation (39). The expression levels of MMP-2 and MMP-9 in colorectal cancer cells are highly related to the metastatic potential $(40,41)$. Herein, we indicated that AITC was involved in MMP-2 and -9 by decreasing their protein level expression (Fig. 3). AITC also enhanced the expression of TIMP-1 level (Fig. 3), leading to the suppression of invasion and migration of EGF-stimulated HT29 cells. These findings also outlined the significance of MMP-2 and -9 in colorectal cancer cell metastasis.

MAPK (ERK, p38 and JNK) signals are known to be correlated with MMP-2 and -9 promoter induction through AP-1 and to regulate the activities of MMP-2/-9 in colorectal cancer cells $(15,42)$. It was reported that inhibition of MAPK/AP-1mediated transcription resulted in reduced migration, invasion, and metastasis in colorectal cancer cells (15). The present study indicated that AITC inhibited the metastasis of EGF-stimulated HT29 cells through reductions of the MAPK signaling pathways in vitro (Fig. 3). 


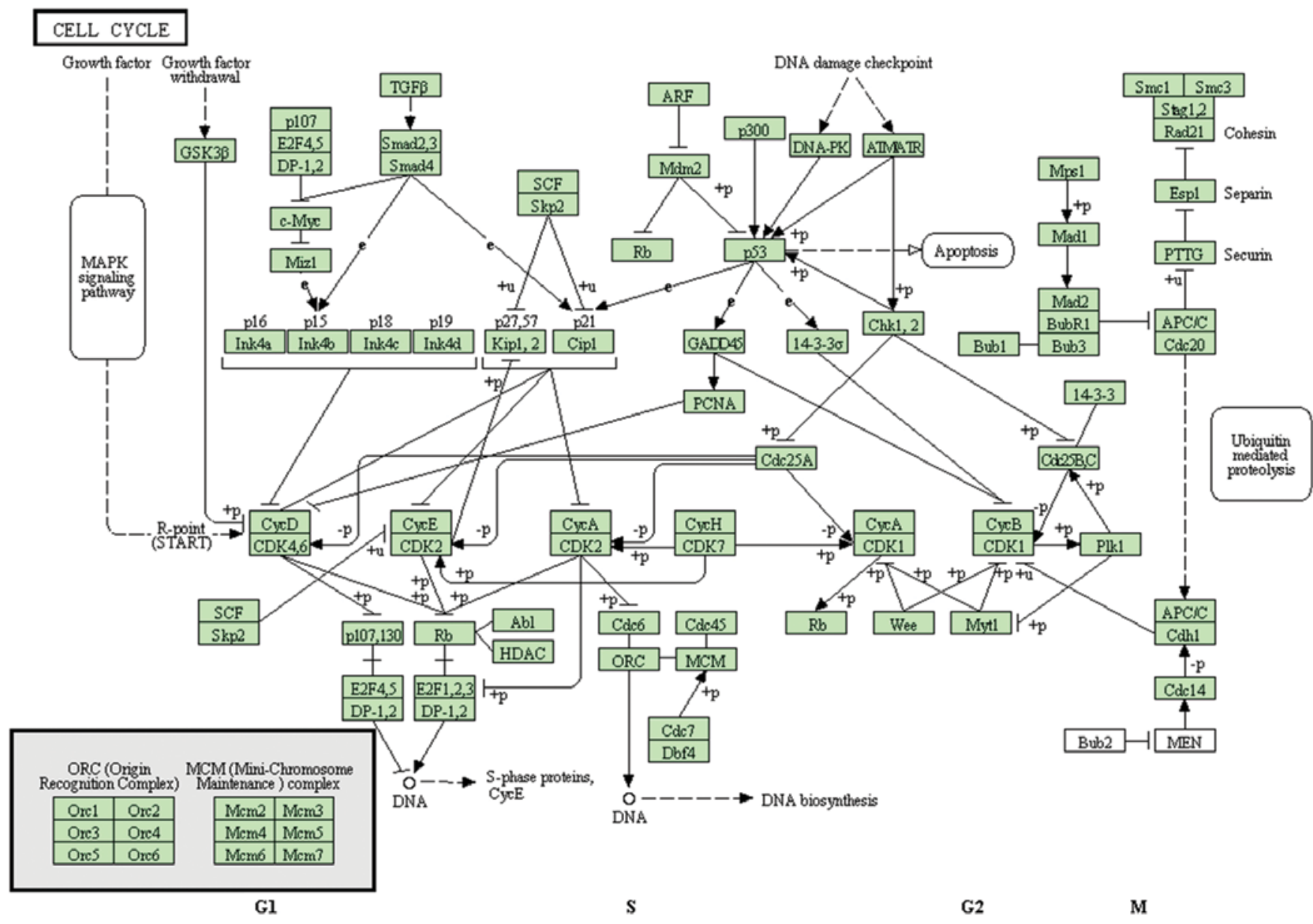

Figure 4. Proposed schematic diagram for the mechanisms of AITC-affected signaling pathways in EGF-stimulated HT29 human colorectal adenocarcinoma cells.

In our previous studies, we demonstrated that AITC induced cell cycle G2/M phase arrest in MDA-MB-468 cells and GBM 8401 cells $(8,10)$. In addition, previous evidence reported that AITC also inhibited cell growth of SW620 human colorectal cancer cells (6), HL-60 leukemia cells (4) as well as prostate cancer PC-3 and LNCaP cells (2) through induction of G2/M phase arrest. In the current study, we examined the change of mRNA expression profile in AITC-treated EGF-stimulated HT29 cells by DNA microarray. Our data showed that cellular and molecular responses to AITC treatment were complicated and likely to be mediated through a variety of regulatory pathways (Fig. 4). AITC regulated the expression of important genes that control cell growth, G-protein coupled receptor, angiogenesis, cell adhesion, cell cycle and mitosis, cell migration, cytoskeleton organization, DNA damage and repair as well as transcription and translation (Table I). Regulation of these genes may be responsible for inhibiting the cell metastasis and cell proliferation of EGF-stimulated HT29 cells. However, we also found that AITC altered the expression of negative regulation of protein kinase activity on EGFR and $\mathrm{PKB} / \mathrm{mTOR}$ signaling genes. We suggested that $\mathrm{PKB} / \mathrm{mTOR}$ signaling genes are also the regulators of translation initiation in MMPs. Inhibition of the $\mathrm{PKB} / \mathrm{mTOR}$ pathways might have the potential to prevent EGF-stimulated HT29 cell invasion and migration. The molecular signaling pathways involved in the effects of AITC on EGF-stimulated HT29 cells are summarized in Fig. 4.
In conclusion, this is the first study to demonstrate that AITC inhibits the metastatic potential actions, including invasion and migration of EGF-stimulated HT29 cells. AITC may be a promising agent in the therapy of colorectal cancer and metastasis of the disease.

\section{Acknowledgements}

The present study was supported by a research grant to K-C.L. awarded by China Medical University Beigang Hospital (CMUBH R101-010).

\section{References}

1. Geng F, Tang L, Li Y, et al: Allyl isothiocyanate arrests cancer cells in mitosis, and mitotic arrest in turn leads to apoptosis via Bcl-2 protein phosphorylation. J Biol Chem 286: 32259-32267, 2011.

2. Xiao D, Srivastava SK, Lew KL, et al: Allyl isothiocyanate, a constituent of cruciferous vegetables, inhibits proliferation of human prostate cancer cells by causing $\mathrm{G} 2 / \mathrm{M}$ arrest and inducing apoptosis. Carcinogenesis 24: 891-897, 2003.

3. Wu CL, Huang AC, Yang JS, et al: Benzyl isothiocyanate (BITC) and phenethyl isothiocyanate (PEITC)-mediated generation of reactive oxygen species causes cell cycle arrest and induces apoptosis via activation of caspase-3, mitochondria dysfunction and nitric oxide (NO) in human osteogenic sarcoma U-2 OS cells. J Orthop Res 29: 1199-1209, 2011.

4. Xu K and Thornalley PJ: Studies on the mechanism of the inhibition of human leukaemia cell growth by dietary isothiocyanates and their cysteine adducts in vitro. Biochem Pharmacol 60: 221-231, 2000. 
5. Bhattacharya A, Li Y, Wade KL, Paonessa JD, Fahey JW and Zhang Y: Allyl isothiocyanate-rich mustard seed powder inhibits bladder cancer growth and muscle invasion. Carcinogenesis 31 2105-2110, 2010

6. Lau WS, Chen T and Wong YS: Allyl isothiocyanate induces $\mathrm{G}_{2} / \mathrm{M}$ arrest in human colorectal adenocarcinoma SW620 cells through down-regulation of $\mathrm{Cdc} 25 \mathrm{~B}$ and $\mathrm{Cdc} 25 \mathrm{C}$. Mol Med Rep 3: 1023-1030, 2010.

7. Schaefer EA, Stohr S, Meister M, Aigner A, Gudermann T and Buech TR: Stimulation of the chemosensory TRPA1 cation channel by volatile toxic substances promotes cell survival of small cell lung cancer cells. Biochem Pharmacol 85: 426-438, 2013.

8. Tsai SC, Huang WW, Huang WC, et al: ERK-modulated intrinsic signaling and $\mathrm{G}_{2} / \mathrm{M}$ phase arrest contribute to the induction of apoptotic death by allyl isothiocyanate in MDA-MB-468 human breast adenocarcinoma cells. Int J Oncol 41: 2065-2072, 2012.

9. Bhattacharya A, Li Y, Geng F, Munday R and Zhang Y: The principal urinary metabolite of allyl isothiocyanate, $\mathrm{N}$-acetyl$\mathrm{S}-(\mathrm{N}$-allylthiocarbamoyl)cysteine, inhibits the growth and muscle invasion of bladder cancer. Carcinogenesis 33: 394-398, 2012.

10. Chen NG, Chen KT, Lu CC, et al: Allyl isothiocyanate triggers $\mathrm{G} 2 / \mathrm{M}$ phase arrest and apoptosis in human brain malignant glioma GBM 8401 cells through a mitochondria-dependent pathway. Oncol Rep 24: 449-455, 2010.

11. Louhivuori LM, Bart G, Larsson KP, et al: Differentiation dependent expression of TRPA1 and TRPM8 channels in IMR-32 human neuroblastoma cells. J Cell Physiol 221: 67-74, 2009.

12. Hwang ES and Kim GH: Allyl isothiocyanate influences cell adhesion, migration and metalloproteinase gene expression in SK-Hep1 cells. Exp Biol Med (Maywood) 234: 105-111, 2009.

13. Garcia A, Haza AI, Arranz N, Rafter J and Morales P: Protective effects of isothiocyanates alone or in combination with vitamin $C$ towards N-nitrosodibutylamine or N-nitrosopiperidine-induced oxidative DNA damage in the single-cell gel electrophoresis (SCGE)/HepG2 assay. J Appl Toxicol 28: 196-204, 2008.

14. Srivastava SK, Xiao D, Lew KL, et al: Allyl isothiocyanate, a constituent of cruciferous vegetables, inhibits growth of PC-3 human prostate cancer xenografts in vivo. Carcinogenesis 24 $1665-1670,2003$

15. Xu C, Shen G, Yuan X, et al: ERK and JNK signaling pathways are involved in the regulation of activator protein 1 and cell death elicited by three isothiocyanates in human prostate cancer PC-3 cells. Carcinogenesis 27: 437-445, 2006.

16. Hwang ES and Lee HJ: Allyl isothiocyanate and its $\mathrm{N}$-acetylcysteine conjugate suppress metastasis via inhibition of invasion, migration, and matrix metalloproteinase-2/-9 activities in SK-Hep 1 human hepatoma cells. Exp Biol Med (Maywood) 231: 421-430, 2006

17. Lai KC, Hsu SC, Kuo CL, et al: Phenethyl isothiocyanate inhibited tumor migration and invasion via suppressing multiple signal transduction pathways in human colon cancer HT29 cells. J Agric Food Chem 58: 11148-11155, 2010.

18. Lan YH, Wu YC, Wu KW, et al: Death receptor 5-mediated TNFR family signaling pathways modulate $\gamma$-humulene-induced apoptosis in human colorectal cancer HT29 cells. Oncol Rep 25: 419-424, 2011

19. Lin HJ, Su CC, Lu HF, et al: Curcumin blocks migration and invasion of mouse-rat hybrid retina ganglion cells (N18) through the inhibition of MMP-2, -9, FAK, Rho A and Rock-1 gene expression. Oncol Rep 23: 665-670, 2010.

20. Lu CC, Yang JS, Chiang JH, et al: Inhibition of invasion and migration by newly synthesized quinazolinone MJ-29 in human oral cancer CAL 27 cells through suppression of MMP-2/9 expression and combined down-regulation of MAPK and AKT signaling. Anticancer Res 32: 2895-2903, 2012.

21. Chen YY, Chiang SY, Lin JG, et al: Emodin, aloe-emodin and rhein inhibit migration and invasion in human tongue cancer SCC-4 cells through the inhibition of gene expression of matrix metalloproteinase-9. Int J Oncol 36: 1113-1120, 2010.

22. Hong BH, Wu CH, Yeh CT and Yen GC: Invadopodia-associated proteins blockade as a novel mechanism for 6-shogaol and pterostilbene to reduce breast cancer cell motility and invasion. Mol Nutr Food Res 57: 886-895, 2013.
23. Chiang JH, Yang JS, Ma CY, et al: Danthron, an anthraquinone derivative, induces DNA damage and caspase cascades-mediated apoptosis in SNU-1 human gastric cancer cells through mitochondrial permeability transition pores and Bax-triggered pathways. Chem Res Toxicol 24: 20-29, 2011.

24. Chiang JH, Yang JS, Lu CC, et al: Newly synthesized quinazolinone HMJ-38 suppresses angiogenetic responses and triggers human umbilical vein endothelial cell apoptosis through p53-modulated Fas/death receptor signaling. Toxicol Appl Pharmacol 269: 150-162, 2013.

25. Lu CC, Yang JS, Chiang JH, et al: Novel quinazolinone MJ-29 triggers endoplasmic reticulum stress and intrinsic apoptosis in murine leukemia WEHI-3 cells and inhibits leukemic mice. PLoS One 7: e36831, 2012.

26. Mozaffarieh M, Konieczka K, Hauenstein D, Schoetzau A and Flammer J: Half a pack of cigarettes a day more than doubles DNA breaks in circulating leukocytes. Tob Induc Dis 8: 14, 2010.

27. Jacobs AT and Marnett LJ: HSF1-mediated BAG3 expression attenuates apoptosis in 4-hydroxynonenal-treated colon cancer cells via stabilization of anti-apoptotic Bcl-2 proteins. J Biol Chem 284: 9176-9183, 2009.

28. Liu CY, Yang JS, Huang SM, et al: Smh-3 induces $\mathrm{G}_{2} / \mathrm{M}$ arrest and apoptosis through calcium-mediated endoplasmic reticulum stress and mitochondrial signaling in human hepatocellular carcinoma Hep3B cells. Oncol Rep 29: 751-762, 2013.

29. Wu RS, Yu CS, Liu KC, et al: Citosol (thiamylal sodium) triggers apoptosis and affects gene expressions of murine leukemia RAW 264.7 cells. Hum Exp Toxicol 31: 771-779, 2012

30. Tang YJ, Yang JS, Lin CF, et al: Houttuynia cordata Thunb extract induces apoptosis through mitochondrial-dependent pathway in HT-29 human colon adenocarcinoma cells. Oncol Rep 22: 1051-1056, 2009.

31. Chen HJ, Jiang YL, Lin CM, et al: Dual inhibition of EGFR and c-Met kinase activation by MJ-56 reduces metastasis of HT29 human colorectal cancer cells. Int J Oncol 43: 141-150, 2013.

32. Friedmann B, Caplin M, Hartley JA and Hochhauser D: Modulation of DNA repair in vitro after treatment with chemotherapeutic agents by the epidermal growth factor receptor inhibitor gefitinib (ZD1839). Clin Cancer Res 10: 6476-6486, 2004.

33. Chu E: Clinical colorectal cancer: the epidermal growth factor receptor signaling pathway as a chemotherapeutic target. Clin Colorectal Cancer 2: 202-203, 2003.

34. Lu Z, Jiang G, Blume-Jensen P and Hunter T: Epidermal growth factor-induced tumor cell invasion and metastasis initiated by dephosphorylation and downregulation of focal adhesion kinase. Mol Cell Biol 21: 4016-4031, 2001

35. Toulany M, Baumann $M$ and Rodemann HP: Stimulated PI3K-AKT signaling mediated through ligand or radiationinduced EGFR depends indirectly, but not directly, on constitutive K-Ras activity. Mol Cancer Res 5: 863-872, 2007.

36. Wang Z, Ahmad A, Li Y, Banerjee S, Kong D and Sarkar FH: Forkhead box M1 transcription factor: a novel target for cancer therapy. Cancer Treat Rev 36: 151-156, 2010.

37. Dong J, Ramachandiran S, Tikoo K, Jia Z, Lau SS and Monks TJ: EGFR-independent activation of p38 MAPK and EGFRdependent activation of ERK1/2 are required for ROS-induced renal cell death. Am J Physiol Renal Physiol 287: F1049-F1058, 2004.

38. Lu P, Weaver VM and Werb Z: The extracellular matrix: a dynamic niche in cancer progression. J Cell Biol 196: 395-406, 2012.

39. Hidalgo $\mathrm{M}$ and Eckhardt SG: Development of matrix metalloproteinase inhibitors in cancer therapy. J Natl Cancer Inst 93: 178-193, 2001.

40. Murray D, Morrin M and McDonnell S: Increased invasion and expression of MMP-9 in human colorectal cell lines by a CD44dependent mechanism. Anticancer Res 24: 489-494, 2004.

41. Kapral M, Wawszczyk J, Jurzak M, Dymitruk D and Weglarz L: Evaluation of the expression of metalloproteinases 2 and 9 and their tissue inhibitors in colon cancer cells treated with phytic acid. Acta Pol Pharm 67: 625-629, 2010.

42. Jeong WS, Kim IW, Hu R and Kong AN: Modulation of AP-1 by natural chemopreventive compounds in human colon HT-29 cancer cell line. Pharm Res 21: 649-660, 2004. 